\title{
Investigation of observability property of controlled binary dynamical systems: a logical approach
}

\author{
G A Oparin, V G Bogdanova and A A Pashinin
}

Matrosov Institute for System Dynamics and Control Theory of SB RAS, Lermontov

St. 134, Irkutsk, Russia, 664033

bvg@icc.ru

\begin{abstract}
The property of observability of controlled binary dynamical systems is investigated. A formal definition of the property is given in the language of applied logic of predicates with bounded quantifiers of existence and universality. A Boolean model of the property is built in the form of a quantified Boolean formula accordingly to the Boolean constraints method developed by the authors. This formula satisfies both the logical specification of the property and the equations of the binary system dynamics. Aspects of the proposed approach implementation for the study of the observability property are considered. The technology of checking the feasibility of the property using an applied microservice package is demonstrated in several examples.
\end{abstract}

\section{Introduction}

Binary dynamical systems (BDS) are widely used in bioinformatics [1, 2], cryptography [3, 4], the study of fault tolerance of computer networks [5, 6], and in many other subjects areas. Recently, the BDS study has attracted considerable attention in systems biology. In particular, it is used as a model of genetic regulatory networks [7]. In our research [8], the Boolean constraints method for the qualitative analysis of BDS dynamic properties is proposed. This method is based on the following provisions:

1. Formalization of dynamic properties definitions in the language of predicate logic and the use of bounded quantifiers of existence and universality;

2. Conversion of the logical property formula that includes the equations of the BDS dynamics;

3. Elimination of bounded quantifiers and obtaining a property formula in the applied logic of predicates with unbounded quantifiers.

A model of the dynamic property in the form of a Boolean constraint is obtained using the sequential execution of these three stages. This model has the form of a Boolean equation or quantified Boolean formula $(\mathrm{QBF})$. The verification of the BDS property is reduced to the Boolean satisfiability problem or verifying QBF truth. These problems are solved using modern SAT [9] and QSAT [10] solvers. In recent years, there has been a significant increase in the performance of specialized algorithms for solving SAT and QSAT problems due to using effective heuristics and deep parallelization of the computational process. Therefore, the variables number in the dynamic property model can be thousands. 
The Boolean constraint method is a fairly general method for the qualitative analysis of BDS on a finite time interval. In [8], this method is used for qualitative analysis of autonomous systems. This study aims to use this method for a qualitative analysis of the observability property of controlled BDS.

The article is structured as follows. Section 2 provides a brief overview of the use of dynamic models in solving the observability problem. In Section 3, a mathematical model of a controlled BDS and a problem statement for verifying $k$-observability for this model are presented. In Section 4, a Boolean equation equivalent to the original system and a formal definition of $k$-observability is given. Also, a Boolean model of this property in the form of a quantified Boolean formula is obtained. The tools and model transformations used for the computer solution of the $k$-observability problem are indicated in Section 5. In Section 6, the proposed technology of qualitative analysis of the $k$ observability property for controlled BDS is demonstrated in several examples. The final Section 7 offers the advantages of the proposed method.

\section{Related work}

Observability is one of the fundamental notions in general control theory [11]. In particular, this applies to the BDS control theory. Observability in control theory is a property that determines the possibility of unambiguous recovery of information about the states of a system from a known output on a finite time interval.

In the last decade, many publications have been devoted to the observability property of BDS (Boolean networks). In [7, 12, 13, 14, 15], various definitions and methods for verifying this property have been proposed. In [12-15], the study of the observability property is based on the approach using the semi-tensor product (STP) of matrices [16]. As noted in [12], such an approach has a disadvantage since the dimension of the obtained matrix is $2^{n} \times 2^{n}$. This disadvantage is the computation complexity for a high dimension $n$ of the BDS state vector. In [13], an estimate of the acceptable value of the dimension $n(n<25)$ is given. For testing observability, an approach based on the idea of representing BDS in polynomial form was proposed [7]. As the authors noted, computing a Gröbner basis [17] used in this method leads, in the general case, to double exponential complexity. In particular, for loosely coupled genetic regulatory networks, the method proposed in [7] is applicable for significantly larger dimensions of the state vector of their Boolean models comparing with the STP method.

A comparative analysis of different types of observability is presented in [18]. Checking the observability property has high computational complexity. So the problem of reducing and speeding up enumeration is fundamental for all the proposed methods. Based on the authors' Boolean constraints method, this problem is solved by SAT [9] and QSAT [10] solvers efficiently.

\section{Problem statement}

A nonlinear BDS of the following form is considered:

$$
x^{t+1}=F\left(x^{t}, u^{t}\right), y^{t}=H\left(x^{t}\right),
$$

where $x(t) \in B^{n}$ is the state vector, $B=\{0,1\}, u(t) \in B^{m}$ is the input (control) vector, $y \in B^{l}$ is the output vector, $n, m, l$ are dimensions of state, control, and output vectors, respectively; $t \in T=\{0,1,2, \ldots, k-1\}$ is the discrete time; $F(x, u), H(x)$ are vector functions of logic algebra, called, respectively, the transition and output function $\left(F: B^{n} \times B^{m} \rightarrow B^{n}, H: B^{n} \rightarrow B^{l}\right)$.

The value $k$ in the definition of the set $T$ is assumed to be a predetermined constant. This limitation occurs for the following reason. In a qualitative study of the behavior of the trajectories of system (1), of practical interest is the feasibility of some dynamic property for a fixed, not too large $k$.

For each state $x^{0} \in B^{n}$ called initial state and for any finite sequence of control vector states $u^{*}=\left[u^{0}, u^{1}, \ldots, u^{k-1}\right]$, let us define for the system (1) a trajectory $x\left(t, x^{0}, u^{*}\right)$ and an output function 
$y\left(t, x^{0}, u^{*}\right)$ as finite sequences of states $\left[x^{0}, x^{1}, \ldots, x^{k}\right]$ and $y^{*}=\left[y^{0}, y^{1}, \ldots, y^{k-1}\right]$ from sets $B^{n}$ and $B^{l}$ respectively. In what follows, the sequence $\left[x^{1}, \ldots, x^{k}\right]$ will be denoted by $x^{*}$.

It is necessary to check for system (1) the satisfiability of the $k$-observability property. We use the following definition of this property, one of several definitions given in [19]. For any two different states $x_{0}, \tilde{x}_{0}$, there is an input sequence $u^{*}$ of length $k$ such that the corresponding output sequences do not coincide $\left(y^{*} \neq \tilde{y}^{*}\right)$.

\section{Solution method}

For $k=1$ (only one-step transitions are considered), system (1) with an initial state $x^{0}$ and input action $u^{*}=\left[u^{0}\right]$ is equivalent to one Boolean equation of the following form:

$$
L\left(x^{0}, x^{1}, u^{0}, y^{0}\right)=\bigvee_{i=1}^{n}\left(x_{i}^{1} \oplus F_{i}\left(x^{0}, u^{0}\right)\right) \vee \bigvee_{i=1}^{l}\left(y_{i}^{0} \oplus H_{i}\left(x^{0}\right)\right)=0,
$$

where $x_{i}^{t}, y_{i}^{t}(t=0,1)$ are $i$-th components of vectors $x^{t}, y^{t} ; F_{i}, H_{i}$ are $i$-th components of vector-functions $F$ and $H$; $\oplus$ is the addition modulo- 2 operation.

For multistep transitions $(k>1)$, system (1) is correspondingly equivalent to the following Boolean equation:

$$
\Phi\left(x^{0}, x^{*}, u^{*}, y^{*}\right)=\bigvee_{t=0}^{k-1} L\left(x^{t}, x^{t+1}, u^{t}, y^{t}\right)=0 .
$$

For the initial state $\tilde{x}^{0}$, equation (2) takes the form

$$
\widetilde{\Phi}\left(\tilde{x}^{0}, \tilde{x}^{*}, u^{*}, \tilde{y}^{*}\right)=\bigvee_{t=0}^{k-1} L\left(\tilde{x}^{t}, \tilde{x}^{t+1}, u^{t}, \tilde{y}^{t}\right)=0 .
$$

According to the method of Boolean constraints, we write the formal definition of the $k$ observability of a BDS in the language of applied logic of predicates with bounded quantifiers:

$$
\left(\forall x^{0}, \tilde{x}^{0}: x^{0} \neq \tilde{x}^{0}\right)\left(\exists u^{*}\right)(\exists t \in T) y\left(t, x^{0}, u^{*}\right) \neq y\left(t, \tilde{x}^{0}, u^{*}\right) \text {. }
$$

Let us get rid of the bounded quantifiers of existence and universality and bear in mind the equations of the dynamics of the $\operatorname{BDS}(2,3)$ for various initial conditions. We obtain the following Boolean model of the observability property in the form of a quantified Boolean formula:

$$
\begin{gathered}
\left(\forall x^{0}, \tilde{x}^{0}\right)\left(\exists x^{*}, \tilde{x}^{*}, u^{*}, y^{*}, \tilde{y}^{*}\right)\left(\bar{E}\left(x^{0}, \tilde{x}^{0}\right) \vee \bar{\Phi}\left(x^{0}, x^{*}, u^{*}, y^{*}\right) \wedge,\right. \\
\left.\wedge \overline{\widetilde{\Phi}}\left(\tilde{x}^{0}, \tilde{x}^{*}, u^{*}, \tilde{y}^{*}\right) \wedge\left(\bigvee_{t=0}^{k-1} E\left(y^{t}, \tilde{y}^{t}\right)\right)\right)
\end{gathered}
$$

where the function $E$ with appropriate arguments satisfies the following Boolean constraint:

$$
E\left(z^{1}, z^{2}\right)=\bigvee_{i=1}^{p}\left(z_{i}^{1} \wedge \bar{z}_{i}^{2} \vee \bar{z}_{i}^{1} \wedge z_{i}^{2}\right)=0 .
$$

This constraint is equivalent to the condition of equality of two Boolean vectors $z^{1}$ and $z^{2}$ of the dimension $p$. The total number of subject variables in formula (4) is $2 k(n+m+l)+2 n$.

\section{Some aspects of program implementation}

The implementation of the proposed approach to the qualitative analysis of the considered dynamic property is based on the Boolean constraints method and performed in the form of an applied 
microservices package (AMP) [20]. This AMP was created based on the HPCSOMAS framework [21].

The AMP provides the following tools for automating the solving problems of qualitative analysis and structural-parametric synthesis of BDS (Figure 1):

- Constructing Boolean models of the dynamic properties of autonomous and controlled BDS;

- Solving a separate problem of the qualitative analysis of BDS (checking the feasibility of a dynamic property);

- Solving complex problems of qualitative analysis of BDS, including performing several separate tasks with alternating construction of Boolean models and checking their feasibility;

- Graphic and tabular visualizing of obtained results.

The listed facilities are structured as separate complexes (processors) of the package. Access to the complexes is performed through the user Dew agent [22]. In figure 1, the structural connections of AMP complexes, grouped in these complexes objects and corresponding used layers of knowledge are shown. The conceptual model of AMP, the construction and use of AMP for solving the problems of qualitative analysis of BDS are discussed in detail in [20].

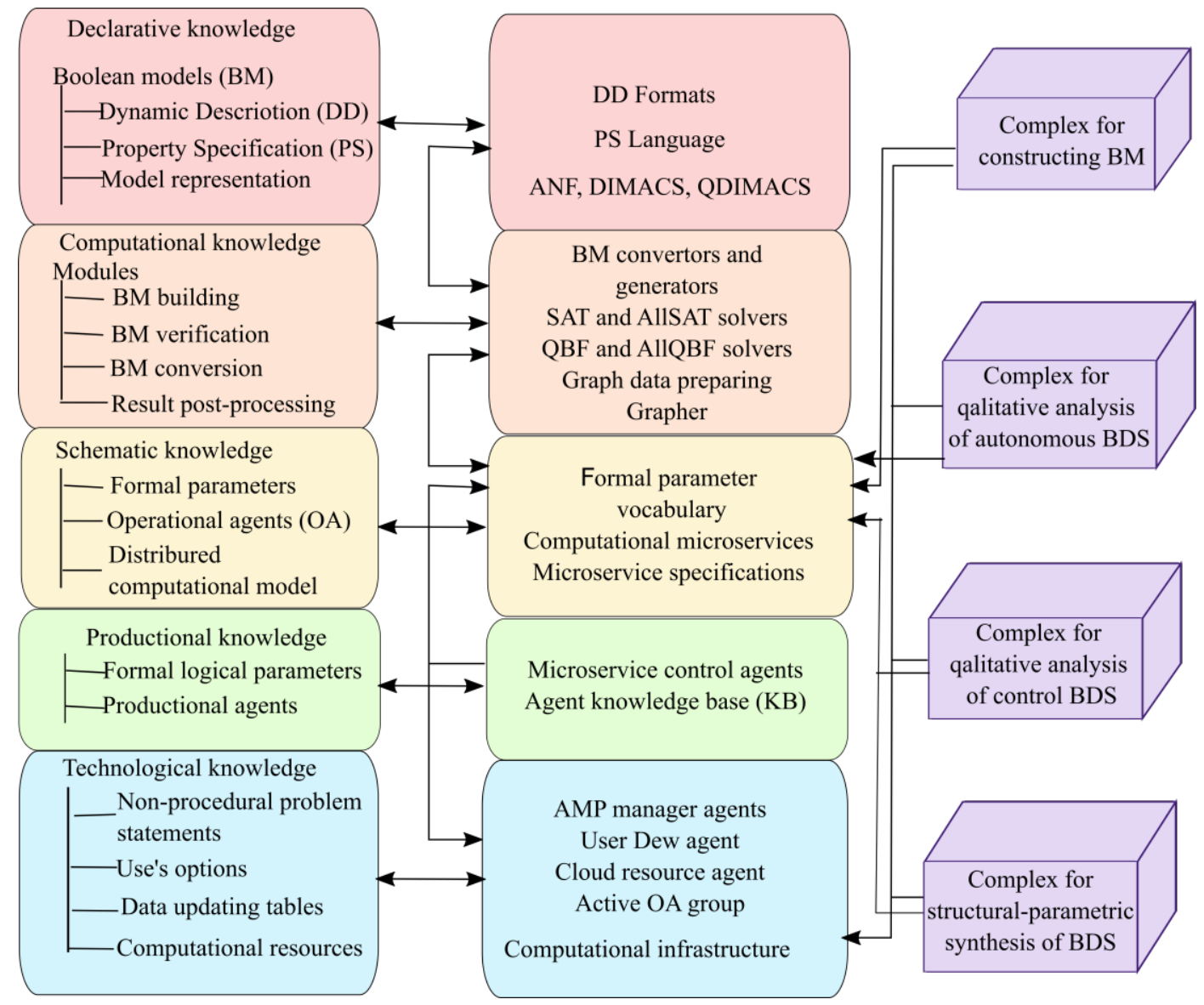

Figure 1. AMP structure.

For checking the observability property, complexes for constructing Boolean models and qualitative analysis of controlled BDS are used. For conversing Boolean expressions in dynamic properties models to CNF, the Tseitin transform [23], the Plaisted-Greenbaum transform [24], and the transformation of the Boolean equation $\mathrm{ANF}=0$ to the form $\mathrm{CNF}=1[25]$ are used. 
For solving Boolean satisfiability problems or checking the QBF truth, computational microservices are used. These microservices are implemented based on the AllSAT solver nbc_minisat_all-1.0.2 [26] and the QSAT solver DepQBF [27]. In the case of a high dimension of the BDS state vector, previously developed parallel solvers $[28,29]$ of a similar purpose are used.

\section{Illustrative example}

The first example shows a detailed computation process.

\subsection{Example 1}

Let us consider the following controlled $\operatorname{BDS}(n=3, m=1, l=1)$ :

$$
\begin{aligned}
& x_{1}^{t+1}=x_{2}^{t} \wedge \bar{x}_{3}^{t} \vee \bar{x}_{2}^{t} \wedge x_{3}^{t} \\
& x_{2}^{t+1}=x_{1}^{t} \wedge \bar{u}^{t} \vee \bar{x}_{1}^{t} \wedge u^{t} \\
& x_{3}^{t+1}=x_{2}^{t} \\
& y^{t}=x_{1}^{t} \wedge \bar{x}_{2}^{t} \wedge \bar{x}_{3}^{t} \vee \bar{x}_{1}^{t} \wedge x_{2}^{t} \wedge \bar{x}_{3}^{t} \vee \bar{x}_{1}^{t} \wedge \bar{x}_{2}^{t} \wedge x_{3}^{t} \vee x_{1}^{t} \wedge x_{2}^{t} \wedge x_{3}^{t}
\end{aligned}
$$

System (5) is equivalent to the following one-step transition equation:

$$
\begin{aligned}
& L\left(x_{1}^{0}, x_{2}^{0}, x_{3}^{0}, x_{1}^{1}, x_{2}^{1}, x_{3}^{1}, u^{0}, y^{0}\right)=x_{1}^{1} \wedge \bar{x}_{2}^{0} \wedge \bar{x}_{3}^{0} \vee \bar{x}_{1}^{1} \wedge x_{2}^{0} \wedge \bar{x}_{3}^{0} \vee x_{1}^{1} \wedge x_{2}^{0} \wedge x_{3}^{0} \vee \bar{x}_{1}^{1} \wedge \bar{x}_{2}^{0} \wedge x_{3}^{0} \vee \\
& \quad \vee x_{2}^{1} \wedge \bar{x}_{1}^{0} \wedge \bar{u}^{0} \vee \bar{x}_{2}^{1} \wedge x_{1}^{0} \wedge \bar{u}^{0} \vee \bar{x}_{2}^{1} \wedge \bar{x}_{1}^{0} \wedge u^{0} \vee x_{2}^{1} \wedge x_{1}^{0} \wedge u^{0} \vee \bar{x}_{3}^{1} \wedge x_{2}^{0} \vee x_{3}^{1} \wedge \bar{x}_{2}^{0} \vee \\
& \quad \vee \bar{x}_{1}^{0} \wedge \bar{x}_{2}^{0} \wedge x_{3}^{0} \wedge \bar{y}^{0} \vee x_{1}^{0} \wedge x_{2}^{0} \wedge x_{3}^{0} \wedge \bar{y}^{0} \vee \bar{x}_{1}^{0} \wedge \bar{x}_{2}^{0} \wedge \bar{x}_{3}^{0} \wedge y^{0} \vee x_{1}^{0} \wedge x_{2}^{0} \wedge \bar{x}_{3}^{0} \wedge y^{0} \vee \\
& \quad \vee x_{1}^{0} \wedge \bar{x}_{2}^{0} \wedge x_{3}^{0} \wedge y^{0} \vee \bar{x}_{1}^{0} \wedge x_{2}^{0} \wedge x_{3}^{0} \wedge y^{0} \vee \bar{x}_{1}^{0} \wedge x_{2}^{0} \wedge \bar{x}_{3}^{0} \wedge \bar{y}^{0} \vee x_{1}^{0} \wedge \bar{x}_{2}^{0} \wedge \bar{x}_{3}^{0} \wedge \bar{y}^{0}=0
\end{aligned}
$$

\begin{tabular}{|c|c|c|c|c|c|c|c|c|c|c|c|c|c|}
\hline Variable & $x_{1}^{0}$ & $x_{2}^{0}$ & $x_{3}^{0}$ & $u^{0}$ & $y^{0}$ & $x_{1}^{1}$ & $x_{2}^{1}$ & $x_{3}^{1}$ & $u^{1}$ & $y^{1}$ & $x_{1}^{2}$ & $x_{2}^{2}$ & $x_{3}^{2}$ \\
\hline Code & 1 & 2 & 3 & 4 & 5 & 6 & 7 & 8 & 9 & 10 & 11 & 12 & 13 \\
\hline Variable & $\tilde{x}_{1}^{0}$ & $\tilde{x}_{2}^{0}$ & $\tilde{x}_{3}^{0}$ & $\tilde{u}^{0}$ & $\tilde{y}^{0}$ & $\tilde{x}_{1}^{1}$ & $\tilde{x}_{2}^{1}$ & $\tilde{x}_{3}^{1}$ & $\tilde{u}^{1}$ & $\tilde{y}^{1}$ & $\tilde{x}_{1}^{2}$ & $\tilde{x}_{2}^{2}$ & $\tilde{x}_{3}^{2}$ \\
\hline Code & 14 & 15 & 16 & 17 & 18 & 19 & 20 & 21 & 22 & 23 & 24 & 25 & 26 \\
\hline
\end{tabular}

To check the property of 3-observability, we write down the Boolean equation of a two-step transition for the initial state $x_{1}^{0}, x_{2}^{0}, x_{3}^{0}$ :

$$
\begin{gathered}
\Phi\left(x_{1}^{0}, x_{2}^{0}, x_{3}^{0}, x_{1}^{1}, x_{2}^{1}, x_{3}^{1}, x_{1}^{2}, x_{2}^{2}, x_{3}^{2}, u^{0}, u^{1}, y^{0}, y^{1}\right)= \\
=L\left(x_{1}^{0}, x_{2}^{0}, x_{3}^{0}, x_{1}^{1}, x_{2}^{1}, x_{3}^{1}, u^{0}, y^{0}\right) \vee L\left(x_{1}^{1}, x_{2}^{1}, x_{3}^{1}, x_{1}^{2}, x_{2}^{2}, x_{3}^{2}, u^{1}, y^{1}\right)=0
\end{gathered} .
$$

The Boolean equation $\tilde{\Phi}=0$ for a two-step transition with the initial state $\tilde{x}_{1}^{0}, \tilde{x}_{2}^{0}, \tilde{x}_{3}^{0}$ is written similarly. In total, expression (4) contains 26 subject variables and 82 clauses. Boolean encoding of subject variables is given in Table 1.

Table 1. Boolean encoding.

When generating Boolean constraints by expression (4), the Plaisted-Greenbaum transform is used. When applying this transformation, two additional variables are introduced. These variables are coded as 27 and 28. 
The expression (4) in QDIMACS format is given in Figure 2.

\begin{tabular}{|c|c|c|}
\hline p cnf 2780 & $-1178-270$ & $\begin{array}{l}-14-15-1618-270 \\
141516-18-270\end{array}$ \\
\hline a 1231415160 & $11-78-270$ & $141516-18-270$ \\
\hline e 45678910 & $-11-7-8-270$ & $-14-15 \quad 16-18-270$ \\
\hline 1112131718 & $117-8-270$ & $-1415-16-18-270$ \\
\hline 1920212223 & $-1269-270$ & $14-15-16-18-270$ \\
\hline 242526270 & $12-69-270$ & $14-1516 \quad 18-270$ \\
\hline $1-14270$ & $126-9-270$ & $-14151618-270$ \\
\hline-114270 & $-12-6-9-270$ & $-242021-270$ \\
\hline $2-15270$ & $13-7-270$ & $24-2021-270$ \\
\hline-215270 & $-137-270$ & $-24-20-21-270$ \\
\hline $3-16270$ & 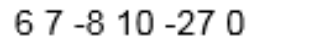 & $2420-21-270$ \\
\hline-316270 & $\begin{array}{llllll}-6 & -7 & -8 & 10 & -27 & 0\end{array}$ & $-251922-270$ \\
\hline$-623-270$ & $678-10-270$ & $25-1922-270$ \\
\hline $6-23-270$ & 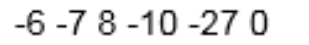 & $2519-22-270$ \\
\hline$-6-2-3-270$ & $-67-8-10-270$ & $-25-19-22-270$ \\
\hline $62-3-270$ & 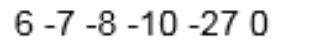 & $26-20-270$ \\
\hline$-714-270$ & 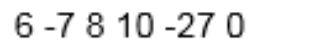 & $-2620-270$ \\
\hline $7-14-270$ & $-67810-270$ & $1920-2123-270$ \\
\hline $71-4-270$ & $-191516-270$ & $-19-20-2123-270$ \\
\hline$-7-1-4-270$ & $19-1516-270$ & $192021-23-270$ \\
\hline $8-2-270$ & $-19-15-16-270$ & $-19-2021-23-270$ \\
\hline$-82-270$ & $1915-16-270$ & $-1920-21-23-270$ \\
\hline $12-3-2750$ & $-2014 \quad 17-270$ & $19-20-21-23-270$ \\
\hline$-1-2-3-2750$ & $20-14 \begin{array}{llll}17 & -27 & 0\end{array}$ & $19-202123-270$ \\
\hline $123-5-270$ & $2014-17-270$ & $-19202123-270$ \\
\hline 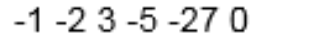 & $-20-14-17-270$ & $-102328-270$ \\
\hline$-12-3-5-270$ & $21-15-270$ & $10-2328-270$ \\
\hline $1-2-3-5-270$ & $-2115-270$ & $\begin{array}{lllll}-4 & 17 & -28 & -27 & 0\end{array}$ \\
\hline $1-235-270$ & $1415-1618-270$ & $4-17-28-270$ \\
\hline
\end{tabular}

Figure 2. QBF for verifying observability property for system (5).

The QBFTV (QBF True Verification) service developed based on the QSAT-solver DepQBF for the quantified Boolean formula (4) returns a message that this formula is TRUE, which means the feasibility of the 3-observability property for system (5). The QBFTV service interface is shown in Figure 3. The QBF in QDIMACS format is used as the input file. The execution result can be viewed on the "Results" tab.

\subsection{Example 2}

Let us consider the Boolean model of the controlled system from [19] $(n=10, m=3, l=7)$ :

$$
\begin{array}{ll}
x_{1}^{t+1}=\bar{x}_{3}^{t} \wedge x_{7}^{t} \wedge \bar{x}_{8}^{t} & x_{8}^{t+1}=x_{3}^{t} \vee \bar{x}_{4}^{t} \wedge \bar{x}_{9}^{t} \\
x_{2}^{t+1}=x_{1}^{t} & x_{9}^{t+1}=x_{5}^{t} \wedge x_{10}^{t} \\
x_{3}^{t+1}=\bar{x}_{4}^{t} \wedge \bar{x}_{9}^{t} & x_{10}^{t+1}=\bar{u}_{1}^{t} \wedge\left(u_{2}^{t} \vee u_{3}^{t} \wedge x_{6}^{t}\right) \\
x_{4}^{t+1}=x_{2}^{t} \wedge x_{5}^{t} & y_{i}^{t}=x_{i}^{t}, i=1,2,6,7 \\
x_{5}^{t+1}=\bar{u}_{1}^{t} \wedge u_{2}^{t} \wedge x_{6}^{t} & y_{3}^{t}=x_{3}^{t} \wedge x_{8}^{t} \\
x_{6}^{t+1}=x_{1}^{t} & y_{4}^{t}=x_{4}^{t} \vee x_{9}^{t} \\
x_{7}^{t+1}=\bar{u}_{1}^{t} & y_{5}^{t}=x_{5}^{t} \wedge x_{10}^{t}
\end{array}
$$




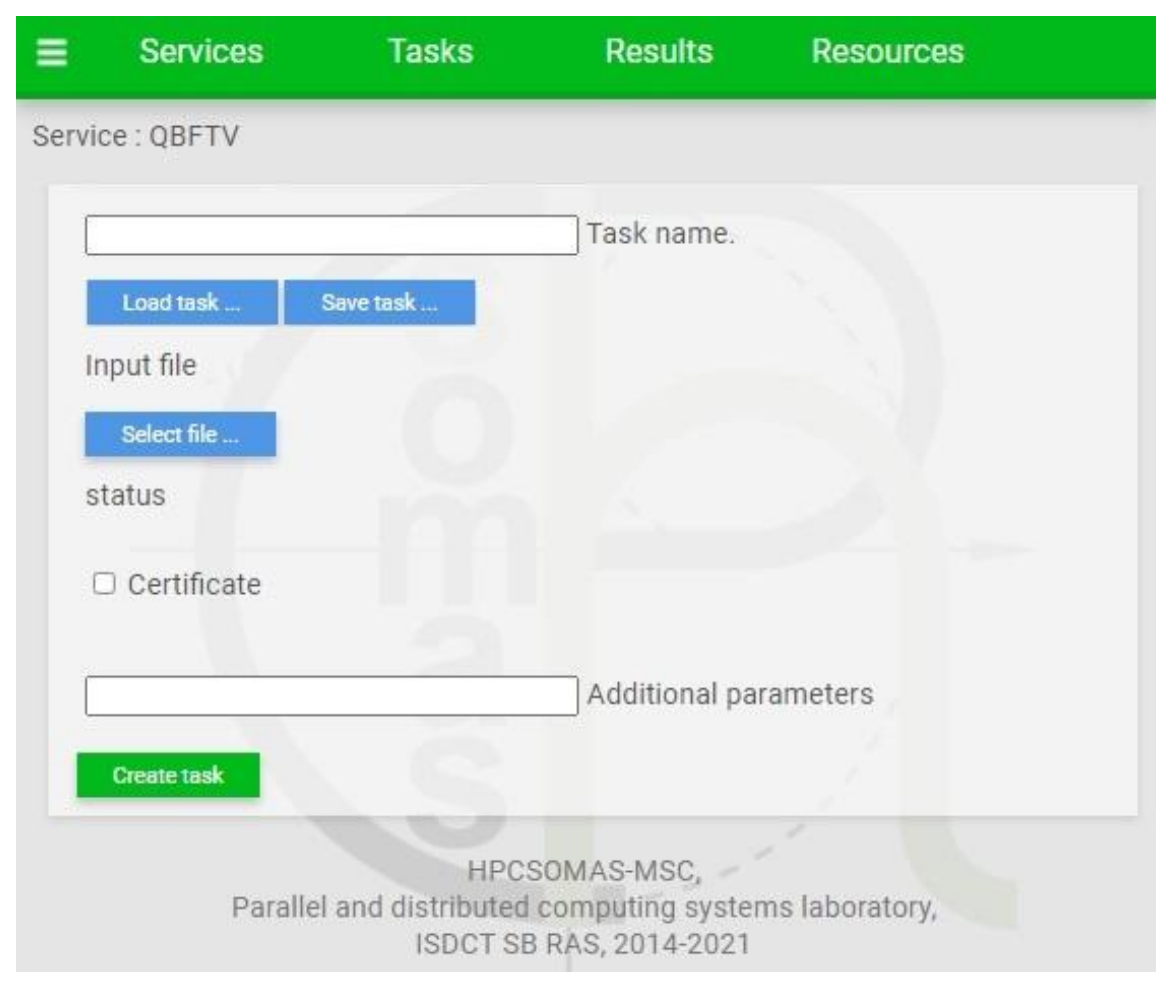

Figure 3. QBFTV service interface.

The one-step transition equation for system (6) has the following form:

$$
\begin{aligned}
& L\left(x_{1}^{0}, x_{2}^{0}, \ldots, x_{10}^{0}, x_{1}^{1}, x_{2}^{1}, \ldots, x_{10}^{1}, u_{1}^{0}, u_{2}^{0}, u_{3}^{0}, y_{1}^{0}, y_{2}^{0}, \ldots, y_{7}^{0}\right)=\bar{x}_{1}^{1} \wedge \bar{x}_{3}^{0} \wedge x_{7}^{0} \wedge \bar{x}_{8}^{0} \vee x_{1}^{1} \wedge x_{3}^{0} \vee x_{1}^{1} \wedge \bar{x}_{7}^{0} \vee x_{1}^{1} \wedge \\
& \wedge x_{8}^{0} \vee \bar{x}_{2}^{1} \wedge x_{3}^{0} \vee x_{2}^{1} \wedge \bar{x}_{3}^{0} \vee \bar{x}_{3}^{1} \wedge \bar{x}_{4}^{0} \wedge x_{9}^{0} \vee x_{3}^{1} \wedge x_{4}^{0} \vee x_{3}^{1} \wedge \bar{x}_{9}^{0} \vee \bar{x}_{4}^{1} \wedge x_{2}^{0} \wedge x_{5}^{0} \vee x_{4}^{1} \wedge \bar{x}_{2}^{0} \vee x_{4}^{1} \wedge \bar{x}_{5}^{0} \vee \\
& \vee \bar{x}_{5}^{1} \wedge \bar{u}_{1}^{0} \wedge u_{2}^{0} \wedge x_{6}^{0} \vee x_{5}^{1} \wedge u_{1}^{0} \vee x_{5}^{1} \wedge \bar{u}_{2}^{0} \vee x_{5}^{1} \wedge \bar{x}_{6}^{0} \vee \bar{x}_{6}^{1} \wedge x_{1}^{0} \vee x_{6}^{1} \wedge \bar{x}_{1}^{0} \vee \bar{x}_{7}^{1} \wedge \bar{u}_{1}^{0} \vee x_{7}^{1} \wedge u_{1}^{0} \vee \bar{x}_{8}^{1} \wedge \\
& \wedge x_{3}^{0} \vee \bar{x}_{8}^{1} \wedge \bar{x}_{4}^{0} \wedge \bar{x}_{9}^{0} \vee x_{8}^{1} \wedge \bar{x}_{3}^{0} \wedge x_{4}^{0} \vee x_{8}^{1} \wedge \bar{x}_{3}^{0} \wedge x_{9}^{0} \vee \bar{x}_{9}^{1} \wedge x_{5}^{0} \wedge x_{10}^{0} \vee x_{9}^{1} \wedge \bar{x}_{5}^{0} \vee x_{9}^{1} \wedge \bar{x}_{10}^{0} \vee \bar{x}_{10}^{1} \wedge \bar{u}_{1}^{0} \wedge \\
& \wedge u_{2}^{0} \vee \bar{x}_{10}^{1} \wedge \bar{u}_{1}^{0} \wedge u_{3}^{0} \wedge x_{6}^{0} \vee x_{10}^{1} \wedge u_{1}^{0} \vee x_{10}^{1} \wedge \bar{u}_{2}^{0} \wedge \bar{u}_{3}^{0} \vee x_{10}^{1} \wedge \bar{u}_{2}^{0} \wedge \bar{x}_{6}^{0} \vee \bar{y}_{1}^{0} \wedge x_{1}^{0} \vee y_{1}^{0} \wedge \bar{x}_{1}^{0} \vee \bar{y}_{2}^{0} \wedge x_{2}^{0} \vee \\
& \vee y_{2}^{0} \wedge \bar{x}_{2}^{0} \vee \bar{y}_{3}^{0} \wedge x_{3}^{0} \wedge x_{8}^{0} \vee y_{3}^{0} \wedge \bar{x}_{3}^{0} \vee y_{3}^{0} \wedge \bar{x}_{8}^{0} \vee \bar{y}_{4}^{0} \wedge x_{4}^{0} \vee \bar{y}_{4}^{0} \wedge x_{9}^{0} \vee y_{4}^{0} \wedge \bar{x}_{4}^{0} \wedge \bar{x}_{9}^{0} \vee \bar{y}_{5}^{0} \wedge x_{5}^{0} \wedge x_{10}^{0} \vee \\
& \vee y_{5}^{0} \wedge \bar{x}_{5}^{0} \vee y_{5}^{0} \wedge \bar{x}_{10}^{0} \vee \bar{y}_{6}^{0} \wedge x_{6}^{0} \vee y_{6}^{0} \wedge \bar{x}_{6}^{0} \vee \bar{y}_{7}^{0} \wedge x_{7}^{0} \vee y_{7}^{0} \wedge \bar{x}_{7}^{0}=0
\end{aligned}
$$

To check the property of 3-observability, we write down the Boolean equation of a two-step transition $(k=2)$ for the initial state $x_{1}^{0}, x_{2}^{0}, \ldots, x_{10}^{0}$ :

$$
\begin{gathered}
\Phi\left(x_{1}^{0}, x_{2}^{0}, \ldots, x_{10}^{0}, x_{1}^{1}, x_{2}^{1}, \ldots, x_{10}^{1}, x_{1}^{2}, x_{2}^{2}, \ldots, x_{10}^{2}, u_{1}^{0}, u_{2}^{0}, u_{3}^{0}, u_{1}^{1}, u_{2}^{1}, u_{3}^{1}, y_{1}^{0}, y_{2}^{0}, \ldots, y_{7}^{0}, y_{1}^{1}, y_{2}^{1}, \ldots, y_{7}^{1}\right)= \\
=\bigvee_{t=0}^{k-1} L\left(x_{1}^{t}, x_{2}^{t}, \ldots, x_{10}^{t}, x_{1}^{t+1}, x_{2}^{t+1}, \ldots, x_{10}^{t+1}, u_{1}^{t}, u_{2}^{t}, u_{3}^{t}, y_{1}^{t}, y_{2}^{t}, \ldots, y_{7}^{t}\right)=0
\end{gathered}
$$

The Boolean equation $\widetilde{\Phi}=0$ for a two-step transition with the initial state $\tilde{x}_{1}^{0}, \tilde{x}_{2}^{0}, \ldots, \tilde{x}_{10}^{0}$ is written similarly. In total, expression (4) contains 100 subject variables and 230 clauses.

The QBFTV service for the quantified Boolean formula (4) gives a message that this formula is FALSE, which means that the observability property for system (6) is not satisfied. 


\subsection{Example 3}

Let us consider the Boolean model of the controlled system from [7] $(n=37, m=3, l=4)$ :

\begin{tabular}{|c|c|c|}
\hline$x_{1}^{1}=x_{9}^{0} \wedge x_{18}^{0}$ & $x_{15}^{1}=x_{34}^{0} \wedge x_{37}^{0}$ & $x_{29}^{1}=x_{12}^{0} \vee x_{30}^{0}$ \\
\hline$x_{2}^{1}=x_{14}^{0}$ & $x_{16}^{1}=x_{13}^{0}$ & $x_{30}^{1}=x_{7}^{0} \vee x_{25}^{0}$ \\
\hline$x_{3}^{1}=x_{2}^{0}$ & $x_{17}^{1}=x_{33}^{0}$ & $x_{31}^{1}=x_{20}^{0}$ \\
\hline$x_{4}^{1}=x_{37}^{0}$ & $x_{18}^{1}=x_{17}^{0}$ & $x_{32}^{1}=x_{8}^{0}$ \\
\hline$x_{5}^{1}=x_{6}^{0}$ & $x_{19}^{1}=x_{37}^{0}$ & $x_{33}^{1}=x_{25}^{0}$ \\
\hline$x_{6}^{1}=x_{32}^{0}$ & $x_{20}^{1}=\bar{x}_{24}^{0} \wedge u_{1}^{0} \wedge u_{2}^{0}$ & $x_{34}^{1}=x_{11}^{0}$ \\
\hline$x_{7}^{1}=x_{26}^{0}$ & $x_{21}^{1}=x_{28}^{0}$ & $x_{35}^{1}=\bar{x}_{4}^{0} \wedge u_{3}^{0}$ \\
\hline$x_{8}^{1}=x_{21}^{0}$ & $x_{22}^{1}=x_{3}^{0}$ & $x_{36}^{1}=x_{10}^{0} \vee\left(x_{20}^{0} \wedge x_{27}^{0}\right)$ \\
\hline$x_{9}^{1}=x_{8}^{0}$ & $x_{23}^{1}=\bar{x}_{16}^{0}$ & $x_{37}^{1}=\bar{x}_{4}^{0} \wedge x_{20}^{0} \wedge x_{36}^{0}$ \\
\hline$x_{10}^{1}=x_{20}^{0} \wedge u_{2}^{0} \vee x_{35}^{0} \wedge u_{2}^{0}$ & $x_{24}^{1}=x_{10}^{0} \wedge \bar{x}_{35}^{0}$ & $y_{1}^{0}=x_{1}^{0}$ \\
\hline$x_{11}^{1}=x_{19}^{0}$ & $x_{25}^{1}=x_{7}^{0}$ & $y_{2}^{0}=x_{5}^{0}$ \\
\hline$x_{12}^{1}=x_{19}^{0}$ & $x_{26}^{1}=x_{27}^{0} \wedge x_{34}^{0} \wedge x_{37}^{0} \wedge\left(x_{15}^{0} \vee x_{31}^{0}\right)$ & $y_{3}^{0}=x_{22}^{0}$ \\
\hline$x_{13}^{1}=x_{25}^{0}$ & $x_{27}^{1}=x_{19}^{0}$ & $y_{4}^{0}=x_{23}^{0}$ \\
\hline$x_{14}^{1}=x_{26}^{0}$ & $x_{28}^{1}=x_{29}^{0}$ & \\
\hline
\end{tabular}

The one-step transition equation for system (7) has the following form:

$$
\begin{aligned}
& L\left(x_{1}^{0}, x_{2}^{0}, \ldots, x_{37}^{0}, x_{1}^{1}, x_{2}^{1}, \ldots, x_{37}^{1}, u_{1}^{0}, u_{2}^{0}, u_{3}^{0}, y_{1}^{0}, y_{2}^{0}, y_{3}^{0}, y_{4}^{0}\right)=\bar{x}_{1}^{1} \wedge x_{9}^{0} \wedge x_{18}^{0} \vee x_{1}^{1} \wedge \bar{x}_{9}^{0} \vee x_{1}^{1} \wedge \bar{x}_{18}^{0} \vee \bar{x}_{2}^{1} \wedge \\
& \wedge x_{14}^{0} \vee x_{2}^{1} \wedge \bar{x}_{14}^{0} \vee \bar{x}_{3}^{1} \wedge x_{2}^{0} \vee x_{3}^{1} \wedge \bar{x}_{2}^{0} \vee \bar{x}_{4}^{1} \wedge x_{37}^{0} \vee x_{4}^{1} \wedge \bar{x}_{37}^{0} \vee \bar{x}_{5}^{1} \wedge x_{6}^{0} \vee x_{5}^{1} \wedge \bar{x}_{6}^{0} \vee \bar{x}_{6}^{1} \wedge x_{32}^{0} \vee x_{6}^{1} \wedge \\
& \wedge \bar{x}_{32}^{0} \vee \bar{x}_{7}^{1} \wedge x_{26}^{0} \vee x_{7}^{1} \wedge \bar{x}_{26}^{0} \vee \bar{x}_{8}^{1} \wedge x_{21}^{0} \vee x_{8}^{1} \wedge \bar{x}_{21}^{0} \vee \bar{x}_{9}^{1} \wedge x_{8}^{0} \vee x_{9}^{1} \wedge \bar{x}_{8}^{0} \vee \bar{x}_{10}^{1} \wedge x_{20}^{0} \wedge u_{2}^{0} \vee \bar{x}_{10}^{1} \wedge x_{35}^{0} \wedge \\
& \wedge u_{2}^{0} \vee x_{10}^{1} \wedge \bar{x}_{20}^{0} \wedge \bar{x}_{35}^{0} \vee x_{10}^{1} \wedge \bar{x}_{35}^{0} \wedge \bar{u}_{2}^{0} \vee x_{10}^{1} \wedge \bar{x}_{20}^{0} \wedge \bar{u}_{2}^{0} \vee x_{10}^{1} \wedge \bar{u}_{2}^{0} \vee \bar{x}_{11}^{1} \wedge x_{19}^{0} \vee x_{11}^{1} \wedge \bar{x}_{19}^{0} \vee \bar{x}_{12}^{1} \wedge \\
& \wedge x_{19}^{0} \vee x_{12}^{1} \wedge \bar{x}_{19}^{0} \vee \bar{x}_{13}^{1} \wedge x_{25}^{0} \vee x_{13}^{1} \wedge \bar{x}_{26}^{0} \vee \bar{x}_{14}^{1} \wedge x_{27}^{0} \vee x_{14}^{1} \wedge \bar{x}_{27}^{0} \vee \bar{x}_{15}^{1} \wedge x_{34}^{0} \wedge x_{37}^{0} \vee x_{15}^{1} \wedge \bar{x}_{34}^{0} \vee x_{15}^{1} \wedge \\
& \wedge \bar{x}_{37}^{0} \vee \bar{x}_{16}^{1} \wedge x_{13}^{0} \vee x_{16}^{1} \wedge \bar{x}_{13}^{0} \vee \bar{x}_{17}^{1} \wedge x_{33}^{0} \vee x_{17}^{1} \wedge \bar{x}_{33}^{0} \vee \bar{x}_{18}^{1} \wedge x_{17}^{0} \vee x_{18}^{1} \wedge \bar{x}_{17}^{0} \vee \bar{x}_{19}^{1} \wedge x_{37}^{0} \vee x_{19}^{1} \wedge \bar{x}_{37}^{0} \vee \\
& \vee \bar{x}_{20}^{1} \wedge \bar{x}_{24}^{0} \wedge u_{1}^{0} \wedge u_{2}^{0} \vee x_{20}^{1} \wedge x_{24}^{0} \vee x_{20}^{1} \wedge \bar{u}_{1}^{0} \vee x_{20}^{1} \wedge \bar{u}_{2}^{0} \vee \bar{x}_{21}^{1} \wedge x_{28}^{0} \vee x_{21}^{1} \wedge \bar{x}_{28}^{0} \vee \bar{x}_{22}^{1} \wedge x_{3}^{0} \vee x_{22}^{1} \wedge \\
& \wedge \bar{x}_{3}^{0} \vee \bar{x}_{23}^{1} \wedge x_{16}^{0} \vee x_{23}^{1} \wedge \bar{x}_{16}^{0} \vee \bar{x}_{24}^{1} \wedge x_{10}^{0} \wedge \bar{x}_{35}^{0} \vee x_{24}^{1} \wedge \bar{x}_{10}^{0} \vee x_{24}^{1} \wedge x_{35}^{0} \vee \bar{x}_{25}^{1} \wedge x_{7}^{0} \vee x_{25}^{1} \wedge \bar{x}_{7}^{0} \vee \bar{x}_{26}^{1} \wedge \\
& \wedge x_{27}^{0} \wedge x_{34}^{0} \wedge x_{37}^{0} \wedge x_{15}^{0} \vee x_{27}^{0} \wedge x_{34}^{0} \wedge x_{37}^{0} \wedge x_{31}^{0} \vee x_{26}^{1} \wedge \bar{x}_{27}^{0} \vee x_{26}^{1} \wedge \bar{x}_{34}^{0} \vee x_{26}^{1} \wedge \bar{x}_{37}^{0} \vee x_{26}^{1} \wedge \bar{x}_{34}^{0} \vee x_{26}^{1} \wedge \\
& \wedge \bar{x}_{15}^{0} \wedge \bar{x}_{31}^{0} \vee \bar{x}_{27}^{1} \wedge x_{19}^{0} \vee x_{27}^{1} \wedge \bar{x}_{19}^{0} \vee \bar{x}_{28}^{1} \wedge x_{29}^{0} \vee x_{28}^{1} \wedge \bar{x}_{29}^{0} \vee \bar{x}_{29}^{1} \wedge x_{12}^{0} \vee \bar{x}_{29}^{1} \wedge x_{30}^{0} \vee x_{29}^{1} \wedge \bar{x}_{12}^{0} \wedge \bar{x}_{30}^{0} \wedge \\
& \wedge \vee \bar{x}_{30}^{1} \wedge x_{7}^{0} \vee \bar{x}_{30}^{1} \wedge x_{25}^{0} \vee x_{30}^{1} \wedge \bar{x}_{7}^{0} \wedge \bar{x}_{25}^{0} \vee \bar{x}_{31}^{1} \wedge x_{20}^{0} \vee x_{31}^{1} \wedge \bar{x}_{20}^{0} \vee \bar{x}_{32}^{1} \wedge x_{8}^{0} \vee x_{32}^{1} \wedge \bar{x}_{8}^{0} \vee \bar{x}_{33}^{1} \wedge x_{25}^{0} \vee \\
& \vee x_{33}^{1} \wedge \bar{x}_{25}^{0} \vee \bar{x}_{34}^{1} \wedge x_{11}^{0} \vee x_{34}^{1} \wedge \bar{x}_{11}^{0} \vee \bar{x}_{35}^{1} \wedge \bar{x}_{4}^{0} \wedge u_{3}^{0} \vee x_{35}^{1} \wedge x_{4}^{0} \vee x_{35}^{1} \wedge \bar{u}_{3}^{0} \vee \bar{x}_{36}^{1} \wedge x_{10}^{0} \vee \bar{x}_{36}^{1} \wedge x_{20}^{0} \wedge \\
& \wedge x_{27}^{0} \vee x_{36}^{1} \wedge \bar{x}_{10}^{0} \wedge \bar{x}_{20}^{0} \vee x_{36}^{1} \wedge \bar{x}_{10}^{0} \wedge \bar{x}_{27}^{0} \vee \bar{x}_{37}^{1} \wedge \bar{x}_{4}^{0} \wedge x_{20}^{0} \wedge x_{36}^{0} \vee x_{37}^{1} \wedge x_{4}^{0} \vee x_{37}^{1} \wedge \bar{x}_{20}^{0} \vee x_{37}^{1} \wedge \\
& \wedge \bar{x}_{36}^{0} \vee \bar{y}_{1}^{0} \wedge x_{1}^{0} \vee y_{1}^{0} \wedge \bar{x}_{1}^{0} \vee \bar{y}_{2}^{0} \wedge x_{5}^{0} \vee y_{2}^{0} \wedge \bar{x}_{5}^{0} \vee \bar{y}_{3}^{0} \wedge x_{22}^{0} \vee y_{3}^{0} \wedge \bar{x}_{22}^{0} \vee \bar{y}_{4}^{0} \wedge x_{23}^{0} \vee y_{4}^{0} \wedge \bar{x}_{23}^{0}=0
\end{aligned}
$$

To check the property of 3-observability, we write down the Boolean equation of a two-step transition $(k=2)$ for the initial state $x_{1}^{0}, x_{2}^{0}, \ldots, x_{37}^{0}$ : 


$$
\begin{gathered}
\Phi\left(x_{1}^{0}, x_{2}^{0}, \ldots, x_{37}^{0}, x_{1}^{1}, x_{2}^{1}, \ldots, x_{37}^{1}, x_{1}^{2}, x_{2}^{2}, \ldots, x_{37}^{2}, u_{1}^{0}, u_{2}^{0}, u_{3}^{0}, u_{1}^{1}, u_{2}^{1}, u_{3}^{1}, y_{1}^{0}, y_{2}^{0}, y_{3}^{0}, y_{4}^{0}, y_{1}^{1}, y_{2}^{1}, y_{3}^{1}, y_{4}^{1}\right)= \\
=\bigvee_{t=0}^{k-1} L\left(x_{1}^{t}, x_{2}^{t}, \ldots, x_{37}^{t}, x_{1}^{t+1}, x_{2}^{t+1}, \ldots, x_{37}^{t+1}, u_{1}^{t}, u_{2}^{t}, u_{3}^{t}, y_{1}^{t}, y_{2}^{t}, y_{3}^{t}, y_{4}^{t}\right)=0
\end{gathered}
$$

The Boolean equation $\widetilde{\Phi}=0$ for a two-step transition with the initial state $\tilde{x}_{1}^{0}, \tilde{x}_{2}^{0}, \ldots, \widetilde{x}_{37}^{0}$ is written similarly. In total, expression (4) contains 250 subject variables and 515 clauses. The QBFTV service for the quantified Boolean formula (4) gives a message that this formula is FALSE, which means that the observability property for system (7) is not satisfied.

\section{Conclusion}

The solution to the qualitative study problem of the $k$-observability property of nonlinear BDS on a finite time interval is obtained using the authors' Boolean constraints method. Recently, BDS (Boolean networks) have attracted considerable attention as computational models for genetic and cellular networks. In this article, we consider observability as the feature determining the initial state of the BDS for given input and output sequences of the length $k$ unequivocally. Based on the logic specification of the dynamic property of $k$-observability and the equations of dynamics of a binary system, a feasibility condition for the $k$-observability property is obtained in the form of a quantified Boolean formula. The verification of the truth of this formula can be performed using an efficient QSAT solver. An advantage of the developed tools for checking the $k$-observability property is their orientation to systems with a high dimension of the state, control, and output vectors and a long interval of discrete-time variation. The proposed method implementation allows data parallelism. So high scalability for increasing the number of variables in the Boolean constraint is provided.

\section{Acknowledgments}

The study was supported by the Ministry of Science and Higher Education of the Russian Federation, project «Technologies for the development and analysis of subject-oriented intelligent group control systems in non-deterministic distributed environments».

\section{References}

[1] Taou N S, Corne D W and Lones M A 2016 Evolving Boolean networks for biological control: State space targeting in scale free Boolean networks IEEE Conference on Computational Intelligence in Bioinformatics and Computational Biology (CIBCB) pp 1-6

[2] Helikar T, Kochi N, Konvalina J and Rogers J A 2011 Boolean Modeling of Biochemical Networks The Open Bioinformatics Journal 5 16-25

[3] Zanin M and Pisarchik A N 2011 Boolean Networks for Cryptography and Secure Communication Nonlinear Sci. Lett. B 1(1) 25-32

[4] Dubrova E and Teslenko M 2016 A SAT-Based algorithm for finding short cycles in shift register based stream ciphers IACR Cryptology ePrint Archive p 1068

[5] Dubrova E, Teslenko M and Tenhunen H 2007 A computational model based on Random Boolean Networks 2nd Bio-Inspired Models of Network, Information and Computing Systems pp 24-31

[6] Dubrova E, Teslenko M and Tenhune H 2008 Self-Organization for Fault-Tolerance SelfOrganizing Systems. IWSOS vol 5343 ed K A Hummel and J P G Sterbenz (Berlin, Heidelberg: Springer)

[7] Li R, Yang M and Chu T 2015 Controllability and observability of Boolean networks arising from biology Chaos 25(2) 023104

[8] Oparin G, Bogdanova V and Pashinin A 2019 Qualitative analysis of autonomous synchronous binary dynamic systems MESA 10(3) 407-419

[9] Gong W and Zhou X 2017 A survey of SAT solver AIP Conference Proceedings vol $1836 \mathrm{p}$ 020059

[10] Marin P, Narizzano M, Pulina L, Tacchella A and Giunchiglia E 2016 Twelve years of QBF 
evaluations: QSAT is PSPACE-hard and it show Fundam. Informaticae 149(1-2) pp 133158

[11] Terrell W J 1999 Some Fundamental Control Theory I: Controllability, Observability, and Duality The American Mathematical Monthly 106(8) 705-719

[12] Cheng D and Qi H 2009 Controllability and observability of Boolean control networks Automatica 45(7) 1659-1667

[13] Zhao Y, Qi H and Cheng D 2010 Input-state incidence matrix of Boolean control networks and its applications Sys. Contr. Lett. 59(12) 767-774

[14] Fornasini E and Valcher M 2013 Observability, reconstructibility and state obververs of Boolean control networks IEEE Trans. Aut. Contr. 58 1390-1401

[15] Laschov D, Margaliot M and Even G 2016 Observability of Boolean networks: A graphtheoretic approach Automatica 49 2351-2362

[16] Cheng D 2007 Sime-tensor product of matrices and its applications. A survey Proc. 4th International Congress of Chinese Mathematicians pp 641-668

[17] Bardet M, Faugère J-C and Salvy B 2015 On the complexity of the $F_{5}$ Gröbner basis algorithm Journal of Symbolic Computation 70 49-70

[18] Zhang K and Zhang L 2016 Observability of Boolean control networks: A unified approach based on finite automata IEEE Trans. Aut. Contr. 61(9) 2733-2738

[19] Cheng D, Qi H, Liu T and Wang Y 2016 A note on observability of Boolean control networks Sys. Contr. Lett. 87 76-82

[20] Oparin G A, Bogdanova V G and Pashinin A A 2020 Microservice approach to the qualitative study of attractors of binary dynamic systems based on the Boolean constraint method Proceedings of the 43rd International Convention on Information, Communication and Electronic Technology (MIPRO), Opatija, 2020 pp 1904-1909

[21] Bychkov I V, Oparin G A, Bogdanova V G, Pashinin A A and Gorsky S A 2017 Automation development framework of scalable scientific web applications based on subject domain knowledge Parallel Computing Technologies. PaCT 2017 vol. 10421 ed V Malyshkin (Cham: Springer, LNCS) pp 278-288

[22] Pashinin A and Bogdanova V 2020 Application of user dew agent in hybrid-computing environments Proceedings of the 1st International Workshop on Advanced Information and Computation Technologies and Systems (AICTS'20) (CEUR-WS Proceedings) 2858 pp 135145

[23] Tseytin G S 1983 On the complexity of derivation in propositional calculus Automation of Reasoning. Symbolic Computation (Artificial Intelligence) ed J H Siekmann and G Wrightson (Berlin, Heidelberg: Springer) pp 466-483

[24] Plaisted D A and Greenbaum S 1986 A Structure Preserving Clause Form Translation J. Smbolic Computation 2(3) 293-304

[25] An ANF to CNF Converter using a Dense/Sparse Strategy. [Online]. Available: https://doc.sagemath.org/html/en/reference/sat/sage/sat/converters/polybori.html.

[26] Toda T and Soh T 2016 Implementing efficient all solutions SAT solvers ACM Journal of Experimental Algorithmics 21(1) 1-44

[27] Lonsing F and Biere A 2010 DepQBF: A dependency-aware QBF solver Journal of Satisfiability, Boolean Modeling and Computation. $971-76$

[28] Bogdanova V G, Gorsky S A and Pashinin A A 2020 HPC-based parallel software for solving applied Boolean satisfiability problems in Proc. of the 43rd Int. Convention on Information and Communication Technology, Electronics and Microelectronics (MIPRO), Opatija, 2020 pp 1006-1011

[29] Bogdanova V G and Gorsky S A 2019 Multiagent technology for parallel implementation of Boolean constraint method for qualitative analysis of binary dynamic systems in Proceeding of the 42nd International Convention on Information and Communication Technology, Electronics and Microelectronics (MIPRO) Opatija, 2019 pp 1043-1048 